\title{
Contraception (4)
}

Série animée par Thierry Jouault

\section{Contraception dans le contexte de l'adolescente}

$>$ La demande d'une contraception par l'adolescente permet de plus au praticien d'évaluer et rediscuter avec elle la vaccination contre le papillomavirus (HPV) et la prévention des infections sexuellement transmissibles (IST), mais aussi de détecter les possibles maltraitances que l'adolescente subit. L'optimisation de l'utilisation de la contraception reste l'objectif prioritaire de la consultation. Toutes les méthodes peuvent être proposées. L'adhésion à telle ou telle méthode est améliorée par une information préalable et un choix qui sera personnalisé. En dehors du risque vasculaire de la contraception œstroprogestative, la contraception hormonale a des effets gynécologiques bénéfiques. L'efficacité des méthodes de longue durée d'action est supérieure à celle des méthodes ponctuelles, mais leur tolérance reste imparfaite. Une contraception d'urgence sera prescrite à l'avance, afin de prévoir tout évènement pouvant survenir de façon inopinée. Des mesures de délivrance sont facilitées pour les mineures. <

\section{Les enjeux de la contraception dans le contexte de l'adolescente}

\section{Données épidémiologiques}

Grossesses et interruption volontaire de grossesse chez les adolescentes

Les grossesses des adolescentes sont un problème de santé publique préoccupant, même si leur nombre diminue régulièrement: en $2013,0,7 \%$ des grossesses mettant au monde un enfant vivant auraient concerné les 15-19 ans (soit 16 477) [1]. Le nombre d'interruptions volontaires de grossesses (IVG) chez les mineures diminue également, avec néanmoins des disparités selon l'âge: il est de 6 pour 1000 grossesses chez les 15-17 ans et d'environ 16 pour 1000 , chez les

Vignette (๔ Inserm/Universcience/CNDP/Picta productions-Véronique Kleiner).

\section{Lise Duranteau}

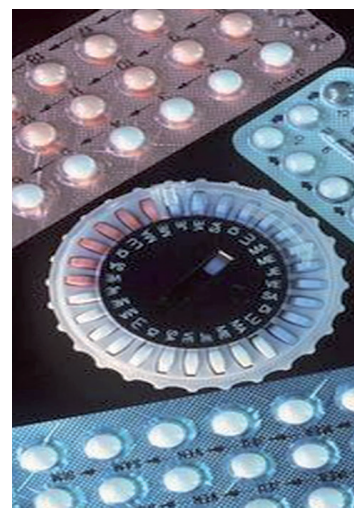

Unité de gynécologie adolescente et jeune adulte, $\mathrm{AP}-\mathrm{HP}$, université Paris Saclay (Bicêtre), Hôpital Bicêtre, 78 rue du Général Leclerc, 94275 Le Kremlin Bicêtre, France. lise.duranteau@aphp.fr

18-19 ans. En revanche, chez les 20-29 ans, le taux d'IVG augmente avec 27,9 IVG pour 1000 grossesses (Figure 1) [2]. Les grossesses donnant au monde un enfant vivant (donc hors IVG) concernent plus souvent des adolescentes issues de milieux défavorisés, ou vivant des situations d'exclusion sociale. La naissance de l'enfant les conduit à interrompre leur scolarité, les rendant ainsi à risque d'isolement et de dépression [1]. Les circonstances dans lesquelles surviennent ces grossesses représentent un frein à la bonne prise en charge des adolescentes, lié à un retard de consultation et à un défaut de leur suivi. Elles expliqueraient une morbidité accrue des mères [3].

\section{Pratiques contraceptives des adolescentes en France}

La contraception orale apparaît être la méthode de contraception la plus utilisée chez les $15-19$ ans (49,6\%), devant le préservatif (25,8\%). Dans $13,3 \%$ des cas, contraception orale et usage du préservatif seraient cependant associés. Le dispositif intra-utérin (DIU) n'est utilisé que par $0,8 \%$ des adolescentes de 18 à 19 ans. Et environ $2 \%$ des adolescentes déclarent ne pas utiliser de contraception (Figure 2) [4]. Des grossesses surviendraient alors que l'adolescente suit une contraception. Pourtant, l'efficacité des méthodes de contraception disponibles rend peu probable l'hypothèse d'un défaut d'efficacité. L'oubli de pilule, l'accident de préservatif et des « erreurs » en cas de méthode locale ou naturelle, peuvent en effet expliquer les échecs de contraception, mais aucune donnée concernant les mineures n'est disponible. Les raisons des grossesses accidentelles chez l'adolescente sont probablement multifactorielles. Améliorer l'utilisation et/ou l'efficacité de la contraception reste donc l'objectif principal dans le contexte de l'adolescente. L'étude française FECOND a révélé que le début précoce d'une contraception, notamment dès le premier rapport sexuel, est le plus souvent associé à 
une utilisation d'une méthode efficace [4]. En France, nous disposons de dispositifs législatifs qui facilitent l'accès à la contraception des mineures, mais l'offre nécessite d'être élargie et doit cibler les adolescentes les moins capables de solliciter une demande auprès du praticien, en particulier celles qui ont un niveau intellectuel bas ou qui sont en situation de vulnérabilité socio-économique, ou dans un contexte familial ou de croyance religieuse qui ne sont pas ouverts à la sexualité et favorables à la contraception [5].

\section{Les enjeux de prévention}

\section{Prévention des infections sexuellement transmissibles}

La consultation pour une contraception doit être le prétexte d'une information sur la prévention des infections sexuellement transmissibles (IST) et leur dépistage. Pour des raisons physiologiques, épidémiologiques et socio-économiques, les adolescentes sont plus vulnérables vis-à-vis des IST. L'existence fréquente d'un ectropion (extériorisation de la zone de jonction squamo-cylindrique du col utérin), accroît leur susceptibilité aux IST. II est également rapporté que, généralement, les adolescentes s'initient sexuellement avec un partenaire plus âgé, avec un usage moindre du préservatif [6]. En raison du caractère peu symptomatique de ces infections et de l'inexpérience des adolescentes, le diagnostic d'IST est tardif. Ce type d'infections peut résulter d'un abus sexuel, qui doit donc être systématiquement envisagé et recherché. Un dépistage régulier est ainsi fortement recommandé chez les adolescentes sexuellement actives. La double protection (contraceptive et contre les IST) doit être encouragée afin que le préservatif ne soit pas abandonné au prétexte de l'utilisation d'un autre mode de contraception. Les utilisatrices de LARC (long-acting reversible contraception), DIU ou implant, seraient plus exposées au risque d'IST. Elles utilisent en effet moins les préservatifs $(60 \%)$ que celles qui prennent un contraceptif oral [6].

\section{Information et prévention des infections à papillomavirus humains (HPV)}

La demande de contraception dans le contexte de l'adolescente est l'opportunité de l'informer sur la prévention des infections par les HPV par la vaccination. La vaccination peut être proposée jusqu'à l'âge de 19 ans, lorsqu'elle n'a pas été réalisée. Son efficacité est établie dans la prévention des infections par les HPV et des condylomes ${ }^{1}$, ainsi que dans la réduction du risque de survenue de lésions pré-cancéreuses de haut-grade et de cancer invasif du col utérin. Cette protection est plus marquée chez les jeunes femmes ayant été vaccinées avant l'âge de 17 ans [7]. Il est néanmoins important de rappeler que la vaccination n'est pas suffisante pour protéger de tous les HPV responsables des cancers du col de l'utérus, et que la stratégie de prévention de ce cancer s'appuie sur une complémentarité entre vaccination et dépistage par prélèvement cervico-utérin (ou frottis).

\footnotetext{
${ }^{1}$ Les condylomes sont des verrues génitales externes bénignes dues à certaines espèces d'HPV.
}

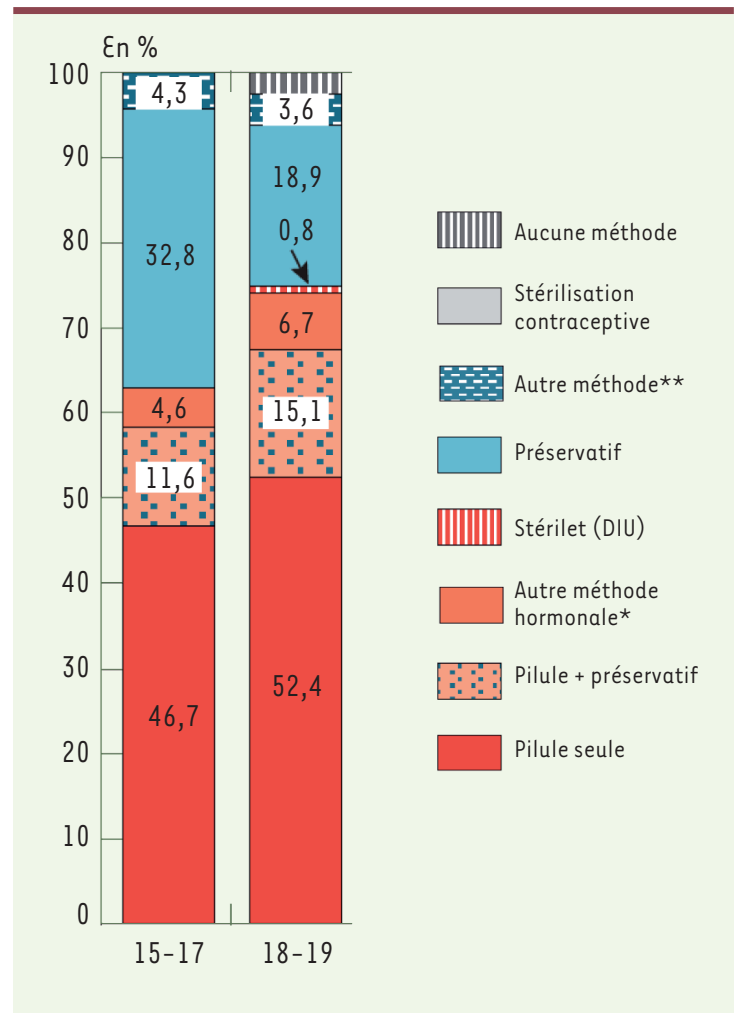

Figure 1. Méthodes de contraception (en \%) utilisées en France en 2016 par les adolescentes âgées de 15-17 ans et de 18-19 ans. *implant, patch, anneau vaginal, ** retrait, abstinence, méthode locale (d'après [4]).

\section{L'abord de la santé sexuelle}

Selon la définition de l'Organisation mondiale de la santé (OMS), la santé sexuelle est un état de bien-être physique, émotionnel, mental et social en matière de sexualité. En France, la première stratégie nationale de santé sexuelle, lancée en 2017, avait pour objectif de promouvoir la santé sexuelle et l'éducation à la sexualité. Une des missions de cette stratégie était de «transformer la consultation longue IST/contraception pour les jeunes filles entre 15 et 18 ans, en une consultation longue 'santé sexuelle' au bénéfice de tous les jeunes ». L'adolescence est une période d'apprentissage de la sexualité. Même si l'âge moyen du premier rapport sexuel en France est de 17,5 ans [8], l'éducation à la sexualité devrait anticiper cette étape, d'autant que les enquêtes sur l'utilisation d'une contraception chez les adolescentes mettent en évidence de nombreux freins à l'accessibilité d'une méthode contraceptive, comme la précarité, le niveau éducatif et la croyance religieuse [8]. Ainsi, les praticiens doivent éduquer les adolescentes au consentement et dépister les situations de maltraitance, les conduites à risque (consommation d'alcool, de drogues) et les éventuels risques psychologiques. 


\section{Les déterminants de la contraception des adolescentes}

\section{Efficacité et observance}

Les contraceptifs disponibles sont très efficaces. En effet, dans leur ensemble, les contraceptifs commercialisés ont une efficacité, exprimée par l'indice de Pearl $(I P)^{2}$, qui est inférieur à l. Les écarts à l'observance sont le principal facteur expliquant les échecs au cours de l'utilisation d'une contraception. Selon James Trussell, l'IP effectif de la contraception hormonale orale, durant la première année d'utilisation, pourrait atteindre 9 (soit 9 grossesses accidentelles sur 100) [9]. Cette évaluation, très utilisée comme référence, a été calculée à partir de données anciennes et provenant d'études aux méthodes différentes. Une autre analyse équivalente, mais excluant les données rétrospectives et antérieures à 1990, fournit des mesures de I'IP plus proches de la réalité, avec, pour la contraception hormonale orale, un IP compris entre 2 et 3 [10]. Les dispositifs intra-utérins (DIU) ou les implants, dont l'efficacité ne dépend pas de l'observance, sont associés à un IP qui est peu modifié en vie réelle [9, 10].

Différents types d'interventions peuvent être proposées pour pallier les défauts d'observance de la contraception orale: 1) I'utilisation facilitée de la contraception d'urgence (par exemple « la pilule du lendemain ») comme méthode de rattrapage en cas d'oubli, sans que cette mesure ne soit associée à une réduction significative des grossesses non prévues; 2) l'utilisation de contraceptifs combinés oraux avec des comprimés placebo (21 comprimés actifs suivis de 7 comprimés placebo) ou la prise en continu de comprimés actifs sans phase d'arrêt. En effet, la mise à disposition de plaquettes comprenant 21 comprimés actifs associés à 7 comprimés placebo entraîne une prise « en continu » sans phase d'arrêt, ce qui serait moins pourvoyeur d'oublis de la reprise ; 3) l'utilisation préférentielle des méthodes contraceptives réversibles de longue durée d'action (ou LARC). Par ces méthodes, une réduction des IVG a en effet été observée aux États-Unis [11]. En France, $2 \%$ des IVG surviendraient avec ce type de contraception, contre $61,5 \%$ avec les autres méthodes, dont $25 \%$ avec la pilule. Pourtant, ces méthodes concernent encore trop peu d'adolescentes (2,8 \% âgées de 15 à 19 ans) [12].

\section{La tolérance}

\section{Le risque vasculaire}

Le risque vasculaire est le principal risque à considérer lors du choix d'une contraception chez une adolescente. Même si le risque absolu d'évènements cardio-vasculaires reste faible, le sur-risque associé à la contraception œstroprogestative, notamment en cas de prédisposition congénitale (thrombophilie), et dans la première année d'utilisation [13], impose le dépistage des facteurs de risque chez les adolescentes dès la première prescription, ou au mieux à l'occasion du premier bilan gynécologique. Les contre-indications relatives, essentiellement l'hypertension artérielle (HTA) non contrôlée, l'obésité,

2 Indice théorique égal au pourcentage de grossesses accidentelles sur un an d'utilisation optimale de la méthode. Un indice de Pearl égal à l signifie que 1 femme sur 100 utilisant la méthode contraceptive analysée pendant un an a été enceinte dans l'année.

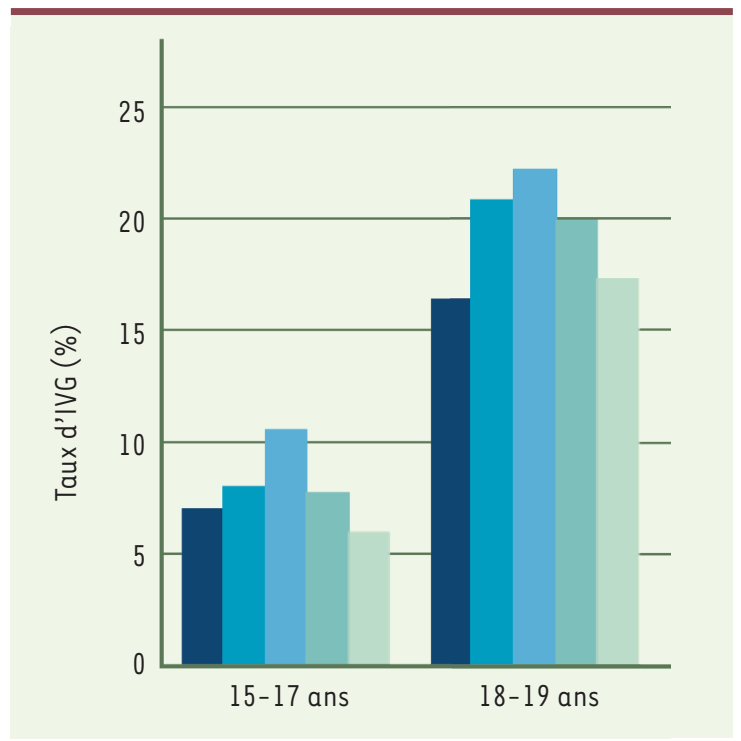

Figure 2. Évolution, entre 1990 à 2019, du taux (en \%) de recours à l'interruption volontaire de grossesse (IVG) chez les adolescentes âgées de 15 à 17 ans et de 18 à 19 ans. Les couleurs représentent les années : du plus foncé au plus clair : 1990, 2000, 2010, 2015, 2019 (d'après [2]).

les migraines, le tabagisme, doivent être également recherchées afin de déterminer la méthode la plus adaptée. Aucun surcroît de risque vasculaire n'est observé pour la contraception progestative, excepté pour la formulation injectable [13].

\section{Les effets indésirables bénins de la contraception hor- monale}

L'utilisation des contraceptifs hormonaux peut être associée à la survenue d'effets indésirables qui se révèlent bénins mais fréquents. II est donc nécessaire d'en informer l'adolescente afin d'éviter tout questionnement ou inquiétude qui seraient à l'origine d'un arrêt prématuré de la prise. Les «fausses-idées » doivent également être corrigées pour obtenir une adhésion de long cours. Les principales craintes concernent:

- les effets indésirables, tels que les nausées, la tension des seins, les troubles du cycle menstruel, les saignements d'intensité modérée. Ces problèmes se résolvent en général spontanément avec la poursuite du traitement ; ils doivent cependant conduire l'adolescente à consulter s'ils persistent ;

- la prise de poids. Elle est souvent associée à la contraception hormonale. Pourtant aucune étude n'a révélé l'existence d'une prise de poids significative [14] ;

- la survenue ou l'exacerbation d'une acné. La contraception hormonale, en particulier œstroprogestative, réduit cependant l'acné préexistant, sans différence entre les différents œstroprogestatifs [15] ; 
- une diminution de la libido. Rapportée au cours d'une contraception hormonale, elle nécessitera d'être abordée afin de rassurer l'adolescente [16].

Les effets indésirables spécifiques des différentes méthodes devront être abordés selon le choix établi en consultation.

\section{Les effets à long terme de la contraception hormonale}

Rassurer sur les effets à long terme de la contraception influe sur l'adhésion de l'adolescente. L'impact sur sa fertilité future est ainsi une crainte forte. En dehors de la contraception injectable, pour laquelle le délai nécessaire pour un retour à des cycles menstruels normaux est retardé, le retour des cycles est immédiat à l'arrêt de la contraception. Pour la contraception œstroprogestative, le taux de grossesse à deux ans est comparable à celui de femmes n'ayant pas eu de contraception [17]. De même, après retrait du DIU, le taux de grossesses à un an est identique à celui de femmes qui n'ont pas utilisé cette méthode [18]. Concernant le risque de cancer, une information objective doit être fournie. Une augmentation du risque de cancer du sein est observée chez les femmes utilisant une contraception hormonale (risque relatif de 1,99 chez celles qui ont débuté leur contraception avant l'âge de 20 ans). Mais le risque absolu de cancer du sein chez les femmes jeunes est faible [19]. En revanche, la contraception hormonale diminue le risque de cancer de l'ovaire (40 à $50 \%$ de réduction) [20] et celui de cancer de l'endomètre [21]. Quant à un éventuel impact sur l'os, le gain de densité minérale osseuse (DMO) est en moyenne plus faible chez les adolescentes prenant une contraception, sans que les conséquences à long terme aient été déterminées [22].

\section{Les bénéfices de la contraception hormonale}

Outre son effet contraceptif, la contraception hormonale [23] présente plusieurs bénéfices pour le traitement de symptômes associés aux cycles menstruels ou celui d'une affection gynécologique. Elle a ainsi un impact immédiat sur la qualité de vie de l'adolescente et influe sur son observance. L'information qui lui sera donnée doit donc intégrer ces bénéfices (Tableau I) :

- le soulagement de la dysménorrhée (ou douleurs menstruelles), lorsqu'elle est sévère et responsable d'absentéisme scolaire [23, 24], ou si elle est associée à une endométriose [25] ;

- la réduction efficace de l'abondance des règles [23] et la prévention du risque de ménorragies (cycles supérieurs à 7 jours) et d'anémie sévère. Les ménorragies seraient, dans au moins $20 \%$ des cas, le premier symptôme révélant un trouble de l'hémostase, qu'il est donc nécessaire de rechercher [26]. Elles sont un facteur aggravant des syndromes anémiques qui sont associés aux hémopathies chroniques. Les contraceptifs hormonaux doivent être proposés pour le contrôle du cycle, au cours d'une maladie chronique, ou en cas de handicap, dans le but d'améliorer la qualité de vie de la femme.

- les œstroprogestatifs sont également recommandés, en première intention, dans le traitement de l'hyperandrogénie, responsable d'acné et de l'hirsutisme, dans le cadre d'une dystrophie ovarienne polykystique [27] ; leur efficacité sur les kystes fonctionnels ovariens reste cependant controversée [28].

\section{Les situations particulières}

Les adolescentes atteintes d'une affection chronique ou neurologique, ou présentant un retard mental, doivent pouvoir bénéficier d'une prévention efficace de la grossesse. En effet, la planification des grossesses chez les femmes présentant une maladie chronique est souvent nécessaire, en raison des risques associés à la maladie elle-même (diabète, maladie inflammatoire, etc.) et/ou à ses traitements (risque tératogène de la dépakine, des immunosuppresseurs). Les adolescentes en situation de handicap sont par ailleurs plus souvent victimes d'abus sexuels [29] susceptibles de provoquer une grossesse.

Ces jeunes filles doivent pouvoir accéder à une consultation avec un gynécologue avant tout besoin contraceptif. Cette consultation permettra d'aborder l'impact que la maladie peut avoir sur la fertilité, de fournir les informations sur les méthodes de contraception les plus adaptées, et sur les interactions médicamenteuses, en accord avec le médecin référent.

\section{Les choix contraceptifs chez l'adolescente}

\section{La contraception hormonale par voie orale}

La contraception combinée œstroprogestative (ou COC) ${ }^{3}$ La contraception œstroprogestative est très efficace avec une bonne observance. Son emploi est facile et elle est souvent très bien tolérée, avec des bénéfices significatifs sur les symptômes associés aux règles, ce qui explique souvent son usage en première intention chez l'adolescente. Le risque vasculaire le plus faible est obtenu avec des associations d'éthinylestradiol et de lévonorgestrel (respectivement 30 et $20 \mu \mathrm{g}$ ) [13]. II est important de souligner que ce risque n'est pas réduit lorsque cette contraception est administrée par une voie non orale. Les contraceptifs de deuxième génération à base de lévonorgestrel, sont donc à privilégier en première intention, à fortiori chez l'adolescente, après avoir écarté les jeunes filles présentant un risque vasculaire.

\section{La contraception progestative par voie orale}

La contraception progestative par voie orale, ou contraception microprogestative, consiste en la prise quotidienne et continue de faibles doses de progestatifs. Le caractère partiel de l'inhibition gonadotrope nécessite une prise en continu pour obtenir l'effet contraceptif. L'aptitude de l'adolescente à une observance rigoureuse

\footnotetext{
${ }^{3}$ Les contraceptifs oraux combinés (COC) sont appelés ainsi car ils contiennent à la fois un estrogène et un progestatif : l'estrogène le plus souvent utilisé est l'éthinylestradiol ; le type de progestatif utilisé varie ; dans le cas d'un progestatif de la classe des norstéroïdes, il détermine la génération de la pilule.
} 


\begin{tabular}{|l|l|}
\hline Symptôme/affection & Bénéfice clinique \\
\hline Contrôle du cycle & $\begin{array}{l}\text { Réduction de la fréquence et de l'abondance des saignements dans } \\
\text { des situations de maladie chronique ou de handicap }\end{array}$ \\
\hline Ménorragies & $\begin{array}{l}\text { Réduction de l'abondance des saignements et réduction du risque } \\
\text { d'anémie en cas de règles abondantes, notamment en cas de mala- } \\
\text { dies de l'hémostase }\end{array}$ \\
\hline Dysménorrhée & Réduction significative de la douleur \\
\hline Endométriose & Réduction du syndrome douloureux \\
\hline Hyperandrogénie & Traitement de l'acné modérée \\
\hline Kystes fonctionnels ovariens & Traitement du syndrome des ovaires polykystiques \\
\hline
\end{tabular}

Tableau I. Synthèse des principaux bénéfices non contraceptifs (en dehors du cancer) liés à l'utilisation de la contraception hormonale orale.

doit donc être évaluée. Cette contraception n'est associée à aucun surcroît de risque vasculaire [13], ce qui facilite sa prescription. Elle est néanmoins à l'origine de troubles du cycle menstruel tels que saignements intercurrents ou aménorrhée. Elle représente donc un avantage si ces effets sont acceptés. Une hypoestrogénie possible (sécheresse vaginale, irritations vulvo-vaginales, voire vulvo-vaginite, diminution de la libido), qui alerte peu l'adolescente, doit être dépistée.

\section{Contraceptions réversibles de longue durée \\ Principes d'utilisation chez l'adolescente}

Les contraceptions réversibles de longue durée d'action (ou LARC pour long-acting reversible contraception) sont les méthodes dont l'efficacité ne dépend pas de l'observance. II s'agit des dispositifs intra-utérins (DIU) au cuivre ou au lévonorgestrel et de l'implant progestatif sous-cutané. Les DIU de petite taille (dits «short » ou « mini » pour les DIU au cuivre) et les modèles de DIU plus faiblement dosés en lévonorgestrel sont plus adaptés à une utilisation par la nullipare. L'excellente efficacité des LARC (Tableau II) permet de réduire significativement et de façon durable les échecs de contraception dans la population adolescente. L'étude prospective CHOICE, réalisée aux États-Unis, a révélé l'efficacité des LARC et leur impact sur la réduction du nombre de grossesses non désirées chez les adolescentes (de 24,4/1 000 grossesses par an). Les taux d'échecs d'une contraception de type LARC, au cours des première, seconde et troisième années d'utilisation, sont respectivement de $0,3 \%, 0,6 \%$ et $0,9 \%$ (contre $4,8 \%, 7,8 \%$ et $9,4 \%$ pour une utilisation d'autres contraceptifs, tels que pilule, patch ou anneau) [11]. Depuis cette étude, les sociétés savantes américaines de pédiatrie et de gynécologie recommandent l'utilisation en première intention de ces méthodes de longue durée d'action chez l'adolescente [30, 31]. En France, leur utilisation reste faible (4,6\% à 6,4 \% d'augmentation en 10 ans [11]) et probablement trop limitée pour observer un impact similaire. Les risques associés à l'utilisation des DIU chez des nullipares participent aux réticences des adolescentes et des praticiens. Les difficultés d'implantation de ces dispositifs seraient plus fréquentes chez ces femmes, comparé aux multipares mais, au total, le DIU est posé avec succès chez les adolescentes âgées de 13 à 17 ans dans $95 \%$ des cas en moyenne, et il n'y a pas de preuve que l'implantation soit plus douloureuse. Le risque de perforation, évalué à 1 à 2 cas sur 1000 , n'a pas été spécifiquement déterminé chez l'adolescente [32]. En France, le choix d'un DIU au cuivre est recommandé en première intention chez la nullipare, en dehors d'une maladie justifiant un DIU hormonal, comme une maladie hémorragique ou un défaut d'hémostase.

La contraception de type injectable, qui consiste en une injection intra-musculaire, tous les 90 jours, d'acétate de médroxyprogestérone, une forme synthétique de progestérone, est peu utilisée en France comme méthode de contraception régulière. C'est néanmoins une méthode de recours de contraception réversible de longue durée d'action, qui peut être utile comme alternative dans des circonstances particulières.

\section{La tolérance}

Les douleurs (observées dans $27 \%$ des cas) et les saignements (dans $21 \%$ des cas) représentent les effets indésirables les plus fréquents de l'utilisation du DIU, et les principales causes de son retrait $[33,34]$. Au long cours, l'utilisation des systèmes intra-utérins libérant un progestatif est associée à une réduction des saignements, voire à une aménorrhée qui peut alors représenter un avantage. L'impact du DIU sur la grossesse, évalué dans l'étude CHOICE, montre que le taux de grossesses un an après son retrait, est comparable à celui d'adolescentes n'ayant pas utilisé de DIU (80\%) (11). En ce qui concerne l'implant, des saignements intercurrents sont rapportés dans $50 \%$ des cas dès les 3 premiers mois d'utilisation. Ce taux diminue ensuite avec $30 \%$ des cas à 6 mois et $20 \%$ à 2 ans. La fréquence 


\begin{tabular}{|c|c|c|c|}
\hline Type de méthode & DIU au cuivre & $\begin{array}{l}\text { DIU au progestatif } \\
\text { (Lévonorgestrel) }\end{array}$ & $\begin{array}{l}\text { Implant progestatif } \\
\text { (Étonogestrel) }\end{array}$ \\
\hline $\begin{array}{l}\text { Efficacité } \\
\text { n grossesses/100 [9, 10] }\end{array}$ & $0,2-1,2 \%$ & $0,1-0,6 \%$ & $0,05-0,2$ \\
\hline Durée d'efficacité & 5 à 10 ans & $\begin{array}{c}3 \text { ans }(13,5 \mathrm{mg}) \\
3 \text { ans }(19,5 \mathrm{mg}) \\
5 \text { ans }(52 \mathrm{mg})\end{array}$ & 3 ans \\
\hline \multicolumn{4}{|c|}{$\begin{array}{l}\text { Taux de poursuite de la méthode } \\
\text { [11] }\end{array}$} \\
\hline $1^{\text {re }}$ année & $86 \%$ & $88 \%$ & $75-83 \%$ \\
\hline $2^{e}$ année & $77 \%$ & $79 \%$ & 70 \% (2,5 années) \\
\hline $3^{e}$ année & $56 \%$ & $52 \%$ & - \\
\hline
\end{tabular}

Tableau II. Les méthodes de contraception progestative de longue durée d'action (LARC) disponibles en France. Efficacité, durée d'action et taux de poursuite de la méthode (d'après [9-11]). DIU : dispositif intra-utérin.

des aménorrhées observées avec ce dispositif est de 30 à $40 \%$ dans les 3 premiers mois, puis de $20 \%$ à 2 ans. Ces troubles sont le motif principal d'arrêt de ce type de contraception [34, 35].

\section{Optimisation de l'utilisation des LARC chez l'adolescente}

L'ensemble des données épidémiologiques sur l'utilisation des contraceptions réversibles de longue durée chez l'adolescente confirme leur efficacité élevée, une tolérance acceptable et un taux de poursuite de la méthode très satisfaisant, ce qui incite à augmenter, voire favoriser, le recours à ces contraceptions dans cette population. II n'existe aucune contre-indication majeure à leur utilisation. L'information donnée aux adolescentes lors de la consultation doit donc valoriser l'efficacité et les avantages de ces dispositifs, en particulier le contrôle du cycle menstruel, de la réduction de l'abondance des règles et de la survenue possible d'aménorrhée en cas d'utilisation de méthodes hormonales. Dans l'étude CHOICE, le taux de poursuite est respectivement de $76 \%, 81 \%$ et $82 \%$, pour le DIU au cuivre, le DIU au lévonorgestrel et l'implant. Le taux de satisfaction s'élève à $75 \%$. En comparaison, le taux d'arrêt des méthodes traditionnelles est de $53 \%$ [11]. L'étude met cependant en évidence le rôle positif de l'accompagnement dans le taux de poursuite de la méthode contraceptive $[11,34]$. L'accès gratuit semble être un facteur majeur du succès de la mise en place et de la poursuite de ces méthodes chez les adolescentes. Leur coût serait également moindre comparé à celui des autres méthodes de contraception (pilule, patch, anneau) [11].

\section{Prescription de la contraception chez l'adolescente}

\section{Les particularités}

\section{Le dispositif législatif}

Des mesures spécifiques ont été mises en place pour faciliter l'accès à la contraception des mineures (Encadré). La gratuité de la consultation, celle des actes utiles à la prescription, des contraceptifs, la délivrance sans prescription, la gratuité de la contraception d'urgence pour les mineures, ainsi que l'assurance de la confidentialité de la prise en charge, représentent des conditions nécessaires pour faciliter l'accès de toutes les adolescentes à la contraception. Ces mesures législatives devraient être connues des prescripteurs, mais aussi de tous les professionnels de santé en contact avec les adolescentes, notamment les infirmiers et les psychologues scolaires, les éducateurs ou les assistantes sociales. Seule la stérilisation n'est pas autorisée chez les mineures.

\section{Le contexte éducatif et psycho-social}

La confidentialité, vis-à-vis de la famille et de l'entourage, doit être garantie à l'adolescente lors de la consultation pour contraception. Une atmosphère de confiance est en effet nécessaire pour obtenir les informations qui sont utiles pour un choix adapté de la méthode qui sera prescrite, comme les habitudes de vie, en particulier scolaires, les activités extra-scolaires, le contexte sociofamilial et la situation de précarité éventuelle. L'activité sexuelle, sa fréquence, le nombre de partenaires, les connaissances sur le risque de grossesse et le risque d'IST, l'utilisation du préservatif, nécessitent d'être évalués. Cette consultation est une opportunité pour rechercher d'éventuelles maltraitances et des conduites à risque addictives ou suicidaires.

\section{La contraception d'urgence chez l'adolescente}

Le DIU posé dans les 5 jours après le rapport à risque est la méthode qui se révèle la plus efficace. Cependant, l'accès au processus en conditions d'urgence est souvent difficile à obtenir, à fortiori pour une adolescente. Les mineures bénéficient de la délivrance gratuite et anonyme de la contraception d'urgence en pharmacie (lévonorgestrel ou acétate d'ulipristal). Elles peuvent également obtenir en milieu scolaire, par l'infirmière scolaire, cette contraception au lévonorgestrel (ou «pilule du 


\section{Les principales mesures législatives relatives à la contraception pour les mineures en France}

1 - Disposition législative relative à la prescription de la contraception en France.

Élargissement des prescripteurs (décret du 10 janvier 2012) : renouvellement pour six mois des contraceptifs hormonaux par les pharmaciens et les infirmières si l'ordonnance médicale date de moins d'un an.

\section{2 - Gratuité des contraceptifs pour les mineures}

2013 : délivrance gratuite des contraceptifs aux mineures de 15 à 17 ans en pharmacie sur prescription (projet de loi de financement de la Sécurité sociale 2013).

2020 : délivrance gratuite des contraceptifs aux mineures de moins de 15 ans (Loi de financement de la Sécurité sociale - article 47)

Gratuité des consultations et des actes de biologie (Loi de santé de 2016):

Consultation annuelle (par un médecin ou une sage-femme) et explorations biologiques dans le cadre de la prescription de la contraception Consultation de suivi (par un médecin ou une sage-femme) au cours de la première année qui suit la prescription de la contraception

Actes liés à la prise en charge, à la pose ou au retrait d'un dispositif contraceptif

Certains examens biologiques (glycémie, cholestérol total, triglycérides) une fois par an si nécessaire

Contraceptifs remboursables

L'ensemble du parcours est protégé par la confidentialité. Si la mineure le demande, il ne sera pas fait mention de son identité ni des actes et des consultations et contraceptifs sur les relevés de remboursement de l'Assurance maladie.

La prescription sur une ordonnance isolée mentionne l'identité, l'âge et les termes: «contraception mineures».

\section{3 - Contraception d'urgence}

Elle est délivrable sans prescription : Lévonorgestrel (en 2000) et acétate d'ulipristal (en 2016)

2002 : gratuité en pharmacie pour les mineures de plus de 15 ans

2020 : délivrance gratuite et anonyme pour toutes les mineures.

Suppression de la condition de « détresse caractérisée » pour son administration par les infirmières scolaires (décret du 26 mai 2016)

lendemain »). Son utilisation optimale nécessite sa prise dans un délai le plus court possible après un rapport à risque. L'intérêt d'une prise précoce pour une efficacité maximale fait proposer une prescription à l'avance de cette contraception d'urgence. La prise combinée d'une contraception progestative et d'acétate d'ulipristal nécessite cependant de protéger les rapports suivants par l'utilisation du préservatif.

\section{Déterminants cliniques chez l'adolescente \\ L'anamnèse $^{4}$}

Le recueil d'informations relatives aux antécédents familiaux et médicaux de l'adolescente qui pourraient contre-indiquer certaines

${ }^{4}$ L'anamnèse est l'ensemble des renseignements fournis au médecin par le malade ou par son entourage sur l'histoire d'une maladie ou les circonstances qui l'ont précédée. méthodes de contraception, comme des antécédents vasculaires ou les caractéristiques de gravité d'une migraine, dans le cas de la contraception œstroprogestative, représente la difficulté essentielle rencontrée par le praticien lors de la première consultation de contraception d'une adolescente. La prise en charge des adolescentes ayant une maladie chronique peut également nécessiter une concertation avec le médecin référent de sa maladie, en cas de traitement pouvant interagir avec la contraception. L'obtention de ces informations est fondamentale, mais ne doit en aucun cas retarder la prescription d'une méthode efficace sans inconvénient médical.

\section{L'examen clinique}

L'anamnèse clinique permet d'orienter le choix de la méthode. Elle doit évaluer le profil des règles, leur fréquence et leur abondance en recherchant des signes de gravité. L'existence de troubles des règles ne doit pas être banalisée : ils doivent être explorés avant la prise de la contraception ou, secondairement, si la contraception doit être débutée rapidement. La dysménorrhée sera recherchée et évaluée pour son intensité, sa durée, la nécessité d'un traitement médicamenteux pour la soulager, et en ce qui concerne son impact sur la vie quotidienne, en particulier sur les activités scolaires ou extra-scolaires. L'existence de signes d'hyperandrogénie et la gêne ressentie seront également évaluées.

L'examen clinique est utile pour relever le poids de référence, évaluer l'état nutritionnel (insuffisance pondérale, surpoids ou obésité). La prise de la pression artérielle est impérative. L'examen des seins est souhaitable afin de dépister toute anomalie avant la prise d'une contraception hormonale. L'examen gynécologique ainsi que l'échographie pelvienne ne sont pas nécessaires en l'absence d'antécédent ou d'élément clinique particulier. En cas de pose de DIU, la recherche préalable d'IST, par des prélèvements locaux (avec recherche des bactéries Chlamydiae trachomatis et Neisseria gonorrhea, en particulier) et sanguins est impérative. $\varepsilon n$ effet, le risque d'infection pelvienne haute à la suite de l'implantation d'un DIU est essentiellement lié à une infection qui est en fait préexistante (33).

\section{Les modalités de prescription et suivi}

Les contre-indications relatives à chaque méthode de contraception ne diffèrent pas entre l'adolescente et la femme plus âgée. En revanche, l'information donnée à l'adolescente doit souligner l'importance de son observance et l'utilisation combinée de préservatifs pour les IST, la possibilité d'un recours à une contraception d'urgence en cas d'oubli, de même que les conditions 
de la gratuité de délivrance en pharmacie et en centre de planification familiale. II faut questionner l'adolescente sur sa capacité d'utiliser des préservatifs, en expliquer la manipulation correcte, et au besoin, la sensibiliser pour associer les garçons à cette prévention, et prescrire des préservatifs qui sont remboursés.

En France, des documents institutionnels édités par l'Institut national de prévention et d'éducation pour la santé (INPES) sont mis à la disposition des professionnels de santé, notamment pour présenter les différentes méthodes de contraception. L'information que ces professionnels donneront doit insister sur la notion de méthode efficace (les méthodes dites «naturelles » ou d'abstinence et de retrait ne sont pas suffisamment efficaces) et sur le choix personnalisé de la méthode qui résultera de la consultation. Elle doit également montrer l'importance d'une observance rigoureuse pour certaines méthodes, en conseillant notamment des stratégies d'aide, par exemple numérique. La contraception peut être immédiatement débutée («quick-start») en associant l'utilisation de préservatifs au cours de la première semaine, mais aussi le plus souvent possible.

Un suivi à court terme sera proposé à l'adolescente pour évaluer sa tolérance vis-à-vis de la méthode choisie, sa bonne compréhension de cette méthode, son observance et la prévention du risque d'IST. Le contrôle de son poids, la mesure de sa pression artérielle, l'évaluation de l'impact de la méthode sur des symptômes gynécologiques possibles, sur sa sexualité et sur sa qualité de vie sont également des paramètres essentiels à considérer lors de cette consultation de suivi. Le changement de méthode contraceptive est rarement nécessaire, mais les difficultés d'observance pourront conduire à rediscuter du choix de la méthode, en soulignant éventuellement l'intérêt des LARC. La consultation de suivi est prise en charge dans le cadre de dispositions législatives spécifiques.

\section{Conclusion}

Selon les données rapportant une utilisation large du préservatif en début de sexualité puis de la contraception orale pour la majorité des adolescentes, et malgré les mesures mises en place pour améliorer l'accès à la contraception des adolescentes, le nombre de grossesses et d'IVG chez les adolescentes ne diminue que peu. Les causes de ce défaut de contraception sont probablement plurifactorielles et complexes, mais les mesures socio-éducatives, pourtant existantes, pourraient être renforcées. Alors que la promotion de l'utilisation de la contraception seule est insuffisante pour obtenir un bénéfice, son association avec des interventions éducatives permet de réduire les grossesses non désirées [36]. En France, les obligations d'éducation à la sexualité et à la contraception pour les établissements scolaires restent cependant insuffisamment satisfaites. Par exemple, les garçons devraient être beaucoup plus associés à cette éducation délivrée essentiellement aux seules adolescentes, au cours des consultations gynécologiques. Des mesures particulières doivent cibler les catégories sociales les plus précaires, qui ont recours à des méthodes dites « naturelles » plutôt qu'aux méthodes qui sont reconnues et efficaces. Certes, la plupart de ces contraceptifs qui sont efficaces nécessitent une prescription médicale, mais ils sont remboursés par l'Assurance maladie.
L'accès à la consultation gynécologique pour une contraception peut poser des problèmes de moyens financiers et de confidentialité. Aussi, une information sur les possibilités de recours aux centres d'éducation et de planification familiale pourrait être optimisée, avec un accès qui serait facilité par leurs horaires et leur répartition territoriale, notamment hors des zones urbaines. La délivrance sans prescription autorisée du lévonorgestrel, dans l'indication de la contraception d'urgence, pourrait être étendue aux contraceptifs progestatifs, avec une durée définie de délivrance. L'enjeu de la gratuité se pose aujourd'hui pour les méthodes les plus efficaces. L'étude prospective américaine $\mathrm{CHOIC \varepsilon} \mathrm{a}$ démontré qu'en offrant aux adolescentes la possibilité d'obtenir gratuitement un moyen de contraception, il était possible d'atteindre un objectif de couverture contraceptive élevée (de $75 \%$ à un an), avec une réduction significative des grossesses de presque $25 \%$ en un an. Cette étude montre également que lorsque le choix leur est laissé, les adolescentes choisissent en majorité des méthodes réversibles de longue durée, avec un taux de poursuite au-delà de la première année élevé [11].

Tous les professionnels impliqués dans l'éducation à la sexualité et la contraception doivent donc être informés de toutes les mesures facilitant l'accès à la contraception pour les adolescentes, mais aussi de l'évolution des connaissances et des pratiques. Les bénéfices potentiels d'amélioration de la qualité de vie doivent aussi être valorisés, en particulier chez les adolescentes en situation de précarité psycho-sociale et chez celles atteintes d'une maladie chronique ou d'un handicap. L'objectif d'une contraception efficace individualisée contribue à l'autonomie des adolescentes dans leur sexualité. $\diamond$

\section{SUMMARY}

\section{Contraception for adolescent women}

The request for a contraception in adolescent women makes it possible to address HPV vaccination and prevention of STIs and to search for abuse. Optimizing the use of contraception is the priority. Any contraceptive methods can be offered. Adhesion is improved by prior information and individualized counselling. Apart from the vascular risk associated with the combined hormonal contraception, hormonal contraception provides gynecologic benefits. The efficacy of long-acting methods is higher compared to shortacting methods but their tolerance is a sensitive matter. Emergency contraception can be prescribed in advance. There are measures to facilitate the delivery to minors. $\diamond$ 


\section{LIENS D'INTÉRÊT}

L'auteure déclare n'avoir aucun lien d'intérêt concernant les données publiées dans cet article.

\section{RÉFÉRENCES}

1. Daguerre A. Les grossesses en France et en Grande-Bretagne. Un phénomène dérangeant pour les pouvoirs publics. Informations Sociales $2010 ; 96-102$.

2. Vilain A. Les interruptions volontaires de grossesse en 2020. Études et Résultats. Paris : DREદS, 2019.

3. Kirbas A, Gulerman HC, Daglar K. Pregnancy in adolescence: is it an obstetrical risk? J Pediatr Adolesc Gynecol $2016 ; 29: 367-71$.

4. Bajos N, Rouzaud-Cornabas M, Panjo H, et al. La crise de la pilule en France : vers un nouveau modèle contraceptif ? Population et Sociétés, $2014 ; 511$.

5. True K, Bajos N, Bohet A, et al. Timing of contraceptive initiation and association with future sexual and reproductive outcomes. Hum Reprod 201429 : 1651-8.

6. Wang J, Roth A, Fang F, et al. HPV vaccination and the risk of invasive cervical cancer. $N$ Engl J Med $2020 ; 383: 1340-8$.

7. Steiner RJ, Liddon N, Swartzendruber AL, et al. Long-acting reversible contraception and condom use among female us high school students: implications for sexually transmitted infection prevention. JAMA Pediatr $2016 ; 170: 428-34$.

8. Jousselme C, Cosquer M, Hassler C. Portraits d'adolescents. Enquête épidémiologique en mileu scolaire en 2013. Paris : Inserm, 2015.

9. Trussell J. Contraceptive failure in the United States. Contraception. $2011 ; 83: 397-404$.

10. Mansour D, Inki P, Gemzell-Danielsson K. Efficacy of contraceptive methods: a review of the literature. Eur J Contracept Reprod Health Care 2010 ; 15 (suppl 2): S19-31.

11. Secura GM, Madden T, McNicholas C, et al. Provision of no-cost, long-acting contraception and teenage pregnancy. $N$ Engl J Med 2014 ; 371 : 1316-23.

12. Moreau C, Bohet A, Hassoun D, et al. Trends and determinants of use of long-acting reversible contraception use among young women in France: results from three national surveys conducted between 2000 and 2010. Fertil Steril $2013 ; 100: 451-8$.

13. Plu-Bureau G, Maitrot-Mantelet L, Hugon-Rodin J, et al. Hormonal contraceptives and venous thromboembolism: an epidemiological update. Best Pract Res Clin Endocrinol Metab 2013 ; 27 : 25-34

14. Gallo MF, Lopez LM, Grimes DA, et al. Combination contraceptives: effects on weight. Cochrane Database Syst Rev $2014 ;$ CD003987.

15. Arowojolu AO, Gallo MF, Lopez LM, et al. Combined oral contraceptive pills for treatment of acne. Cochrane Database Syst Rev 2012 ; CD004425.

16. Malmborg A, Persson $\varepsilon$, Brynhildsen J, et al. Hormonal contraception and sexual desire: a questionnaire-based study of young Swedish women. Eur J Contracept Reprod Health Care 2016; $21: 158-67$.

17. Barnett C, Hagemann C, Dinger J, et al. Fertility and combined oral contraceptives - unintended pregnancies and planned pregnancies following oral contraceptive use - results from the INASSCORE study. Eur J Contracept Reprod Health Care 2017 ; 22 : 17-23.

18. Stoddard AM, Xu H, Madden T, et al. Fertility after intrauterine device removal: a pilot study. Eur J Contracept Reprod Health Care $2015 ; 20: 223-30$.

19. Morch L.S, Skovlund C.W, P.C. Hannaford, et al. Contemporary hormonal contraception and the risk of breast cancer. $N$ Engl J Med 2017 ; 377 : 2228-39.

20. Iversen $L$, Fielding $S$, Lidegaard $\emptyset$, et al. Association between contemporary hormonal contraception and ovarian cancer in women of reproductive age in Denmark: prospective, nationwide cohort study. BMJ $2018 ; 362$ : k3609.
21. Collaborative group on epidemiological studies on endometrial C. Endometrial cancer and oral contraceptives: an individual participant meta-analysis of 27276 women with endometrial cancer from 36 epidemiological studies. Lancet Oncol 2015; 16 : 1061-70.

22. Barchrach L.K. Hormonal contraception and Bone health in adolescents and contraceptives. Front Endocrinol $2020 ; 11: 603$.

23. Bahamondes L, Bahamondes VM, Shulman LP. Non-contraceptive benefits of hormonal and intrauterine reversible contraceptive methods. Hum Reprod Update $2015 ; 21: 640-51$.

24. Davis AR, Westhoff C, O'Connell K, et al. Oral contraceptives for dysmenorrhea in adolescent girls: a randomized trial. Obstet Gynecol 2005 $106: 97-104$

25. Harada T, Momoeda M, Taketani Y, et al. Low-dose oral contraceptive pil for dysmenorrhea associated with endometriosis: a placebo-controlled, double-blind, randomized trial. Fertil Steril $2008 ; 90: 1583-8$.

26. Haamid F, Sass AE, Dietrich JE. Heavy menstrual bleeding in adolescents. J Pediatr Adolesc Gynecol 2017 ; 30 : 335-40.

27. Dokras A. Noncontraceptive use of oral combined hormonal contraceptives in polycystic ovary syndrome-risks versus benefits. Fertil Steril 2016; 106 : 1572-9.

28. Grimes DA, Jones LB, Lopez LM, et al. Oral contraceptives for functional ovarian cysts. Cochrane Database Syst Rev 2014 ; CD006134.

29. Quint $\varepsilon H$. Menstrual and reproductive issues in adolescents with physical and developmental disabilities. Obstet Gynecol $2014 ; 124$ : 367-75.

30. Chabbert-Buffet N, Marret H, Agostini A, et al. Clinical practice guidelines for contraception by the French national college og gynecologists and obstetricians. Gynecol Obstet Hum Reprod 2019 ; 48 : 441-54.

31. Committee on A. Contraception for adolescents. Pediatrics 2014 ; 134 el244-5.

32. Committee on Adolescent Health C. Committee opinion no 699: adolescent pregnancy, contraception, and sexual activity. Obstet Gynecol 2017 ; 129 : el42-e9.

33. Bahamondes MV, Bahamondes L. Intrauterine device use is safe among nulligravidas and adloescent girls. Acta Obstet Gynecol Scand 2021; 100 : 641-8

34. Modesto W, Bahamondes MV, Bahamondes L. A randomized clinical trial of the effect of intensive versus non-intensive counselling on discontinuation rates due to bleeding disturbances of three long-acting reversible contraceptives. Hum Reprod 2014 ; 29 : 1393-908.

35. Darney P, Patel A, Rosen K, et al. Safety and efficacy of a single-rod etonogestrel implant (Implanon): results from 11 international clinical trials. Fertil Steril $2009 ; 91: 1646-53$.

36. Oringanje C, Meremikwu MM, Eko H, et al. Interventions for preventing unintended pregnancies among adolescents. Cochrane Database Syst Rev $2016 ; 2$ : CD0052.

\section{LA FONDATION PREMUP : UN OPÉRATEUR DE TERRAIN EN PÉRINATALITÉ RECONNU POUR SON EXCELLENCE ET SON INTERDISCIPLINARITÉ}

La Fondation de coopération scientifique PremUp, unique en Europe, intervient sur la prévention du handicap à la naissance, par la protection de la santé de la femme enceinte et du nouveau-né.

TIRÉS À PART

L. Duranteau

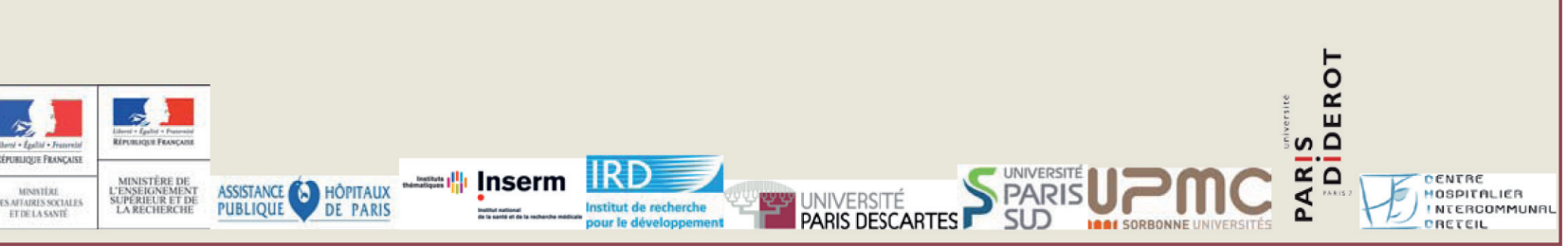

